\title{
OBSTRUÇÃO EM ALÇA FECHADA COM RUPTURA DO CECO: ASPECTOS NA TOMOGRAFIA COMPUTADORIZADA - RELATO DE UM CASO*
}

\author{
Sheila Jandhyra Vianna Crespo ${ }^{1}$, Edson Marchiori², Luis Fernando Mendes ${ }^{3}$
}

Resumo A obstrução intestinal em alça fechada determina uma situação de extrema gravidade, com conseqüências sérias, podendo por vezes levar ao óbito. Os autores descrevem um caso de obstrução intestinal secundária a câncer no sigmóide, destacando a importância da tomografia computadorizada na análise das alterações patológicas na rotina de abdome agudo.

Unitermos: Tomografia computadorizada. Obstrução intestinal. Ruptura do ceco. Câncer de sigmóide.

Abstract Computed tomography findings in closed obstruction of the small bowel associated with rupture of the cecum
- a case report.
Closed loop obstruction of the bowel is an extremely grave condition that may lead to serious consequences
and even be fatal. The authors describe a case of patient with intestinal obstruction secondary to cancer
of the sigmoid and highlight the importance of computed tomography in the analysis of the pathological
changes in routine management of patients with acute abdomen.
Key words: Computed tomography. Small bowel obstruction. Rupture of the cecum. Cancer of the sigmoid.

\section{INTRODUÇÃO}

A obstrução intestinal mecânica aguda constitui uma das causas mais freqüentes de abdome agudo. No cólon descendente, na altura da transição colo-retal, é uma das complicações mais comuns da idade avançada, geralmente representando situação de emergência que implica tratamento cirúrgico imediato ${ }^{(1-6)}$.

O câncer colo-retal é a segunda principal causa de doença maligna no idoso e corresponde a um dos mais altos porcentuais dentre os atendimentos hospitalares de emergência ${ }^{(7-9)}$. As principais etiologias dos processos obstrutivos no intestino grosso são, de modo geral, de naturezas diferentes daquelas do intestino delgado. Enquanto no delgado as obstruções decorrem principalmente da presença de bridas e aderências, no intes-

* Trabalho realizado no Serviço de Radiologia do Instituto Nacional de Câncer (INCA), Rio de Janeiro, RJ.

1. Professora Adjunta de Radiologia da Universidade Federal Fluminense (UFF), Médica do Serviço de Radiologia do INCA/RJ.

2. Professor Titular de Radiologia da UFF, Coordenador Adjunto do Curso de Pós-graduação em Radiologia da Universidade Federal do Rio de Janeiro.

3. Médico Residente do Serviço de Radiologia do INCA/ RJ.

Endereço para correspondência: Prof. Dr. Edson Marchiori. Rua Thomaz Cameron, 438, Valparaíso. Petrópolis, RJ, 25685-120. E-mail: edmarchiori@zipmail.com.br

Aceito para publicação em 4/12/2000. tino grosso costumam ser devidas a infecções ou a processos tumorais ${ }^{(\mathbf{1 , 2})}$.

O exame do tubo digestivo pela tomografia computadorizada vem sendo cada vez mais utilizado, devido à capacidade deste método de imagem de visibilizar tanto a luz como a extensão serosa dos processos patológicos, embora a definição precisa das doenças freqüentemente dependa da correlação com os sintomas clínicos. Nos pacientes com câncer coloretal, o exame tomográfico é muito importante, não só para o estudo da extensão local da doença, como também para avaliar a disseminação metastática ${ }^{(\mathbf{1 0})}$. A precocidade do diagnóstico é fundamental, devendo a terapêutica cirúrgica ser o mais imediata possível, uma vez que a impossibilidade de progressão do conteúdo intestinal determina necrose e perfuração da alça. Complicações do câncer de cólon incluem intussuscepção, obstrução e perfuração, todas elas passíveis de avaliação pela tomografia computadorizada ${ }^{(3,10,11)}$.

O tratamento preconizado requer cirurgia de emergência, geralmente estando o paciente em condições clínicas precárias, o que determina elevadas taxas de mortalidade e morbidade ${ }^{(2,4,9,11,12)}$.

A obstrução do intestino grosso requer colostomia descompressiva para melhora das condições clínicas num pri- meiro estágio, enquanto a ressecção do tumor deverá ocorrer em tempo cirúrgico posterior. Nos pacientes não-candidatos à ressecção tumoral, seja por alto grau de evolução do câncer, alto risco cirúrgico ou por metástases disseminadas, a colostomia descompressiva pode ser a única opção terapêutica ${ }^{(\mathbf{4 , 6})}$.

Em trabalhos recentes da literatura, alguns autores têm sido favoráveis ao tratamento não-cirúrgico da obstrução intestinal, optando pela colocação de "stent" metálico auto-expansível para manter a permeabilidade do segmento de alça estenosado pelo tumor, prevenindo, assim, a obstrução total ${ }^{(4-6,9,13)}$.

\section{RELATO DO CASO}

Paciente do sexo masculino, 64 anos de idade, com adenocarcinoma do sigmóide previamente conhecido, foi atendido pelo Serviço de Emergência do Instituto Nacional de Câncer/RJ, com quadro de abdome agudo. Ao exame físico, apresentava dor, distensão abdominal, parada de eliminação de gases e fezes, sinais de desidratação, dispnéia e fraqueza muscular.

O exame pela tomografia computadorizada (Figura 1) demonstrou acentuada distensão dos cólons transverso e ascendente e do ceco. O cólon ascendente 


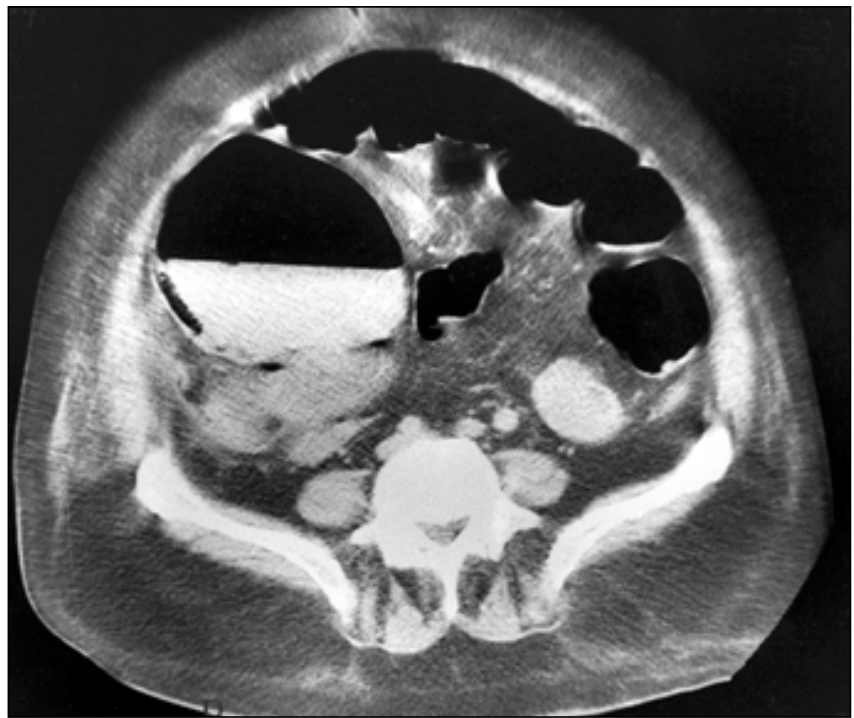

A

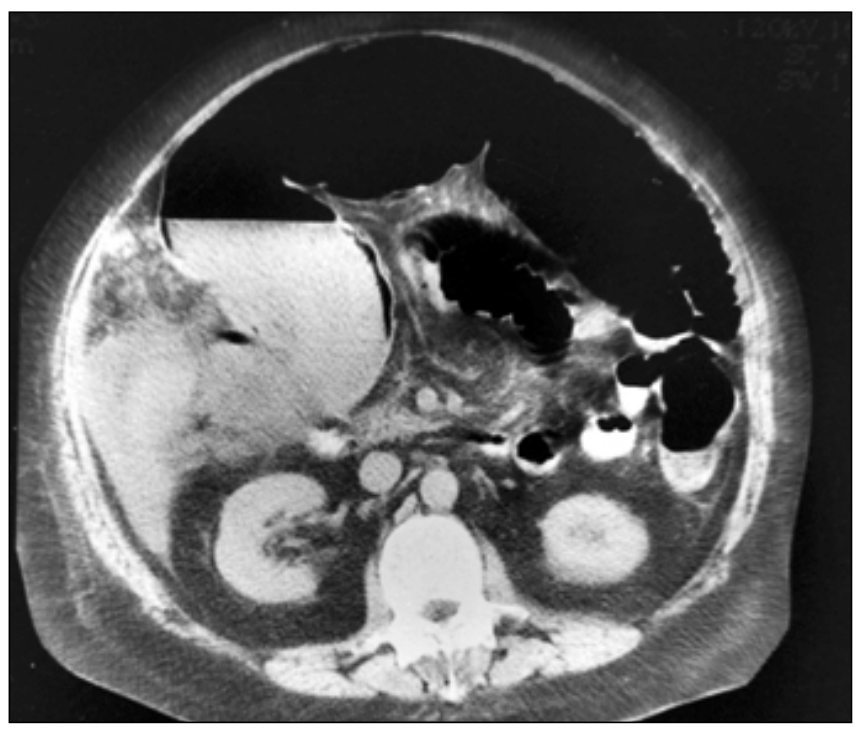

C

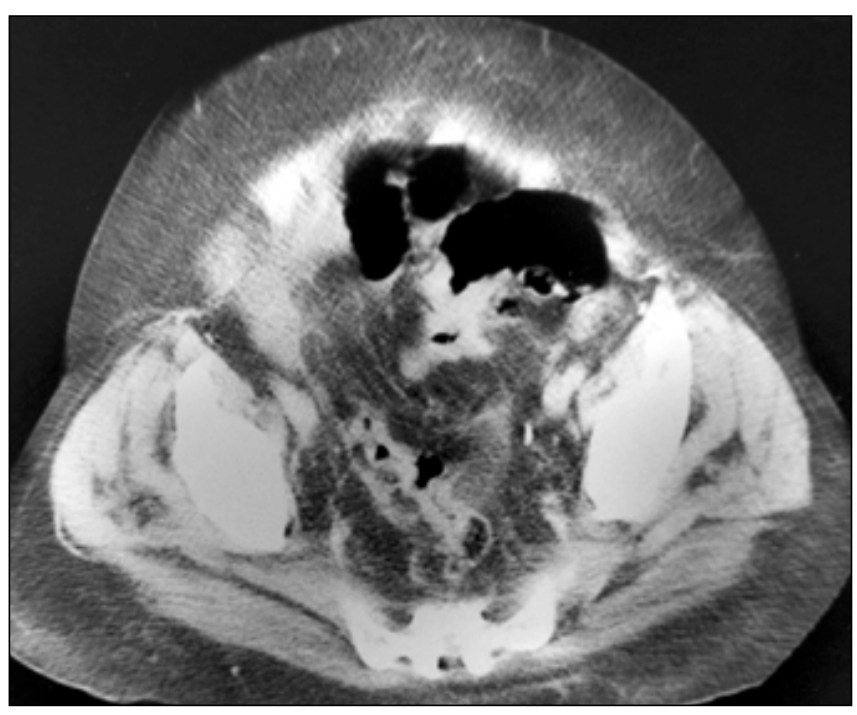

$\mathbf{E}$

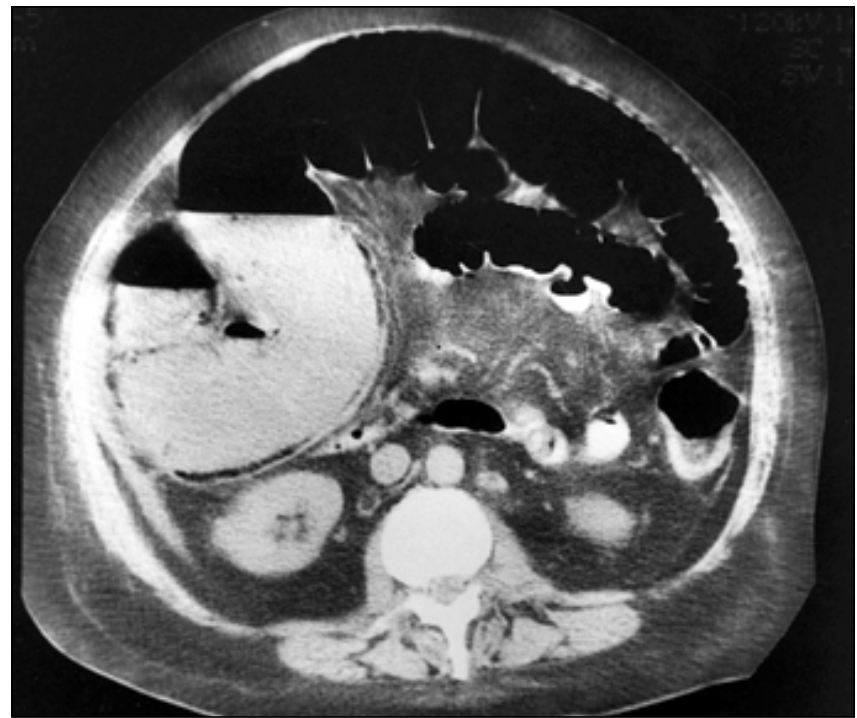

B

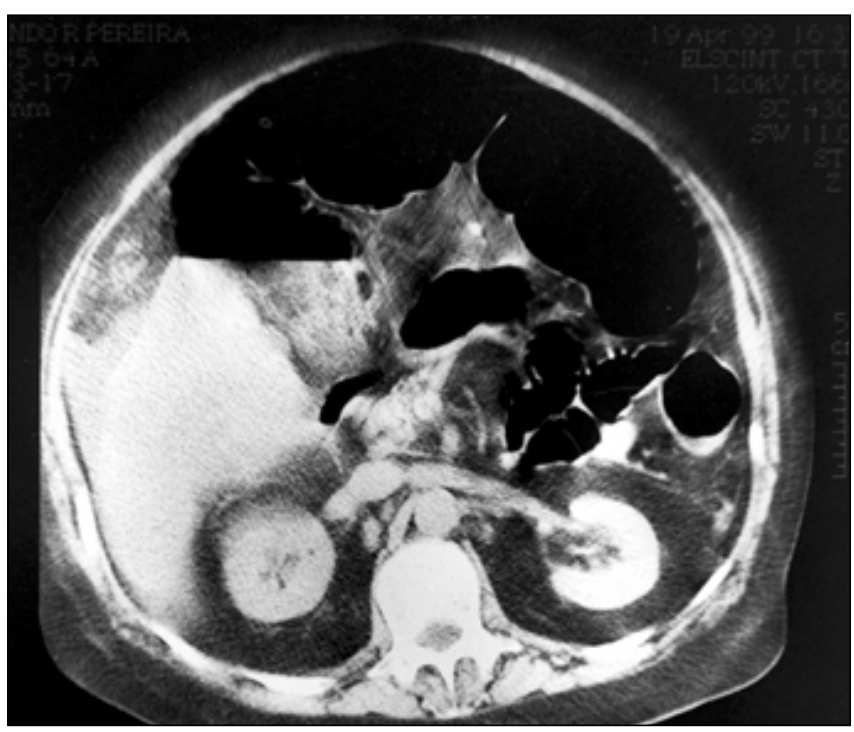

D

Figura 1. Tumor de sigmóide com ruptura do ceco. Tomografia computadorizada do abdome com uso de meio de contraste venoso demonstrando alças do cólon ascendente e ceco dilatadas, com níveis líquidos espontaneamente densos, por conteúdo hemorrágico, além de sinais de dissecção parietal gasosa. Em A, detalhe do calibre do ceco, com aproximadamente $13 \mathrm{~cm}$ de diâmetro; em $\mathbf{B}$ e $\mathbf{C}$ observa-se presença de conteúdo hemorrágico na goteira parietocólica direita, e em D, volumosa coleção hemorrágica no espaço pararrenal anterior. Em E, nota-se acentuada redução de calibre da alça intestinal na altura do sigmóide, com dilatação gasosa de alças a montante. 
e o ceco apresentavam líquido espontaneamente denso, configurando conteúdo hemorrágico, além de sinais de dissecção parietal gasosa. Havia também coleção hemorrágica no espaço pararrenal anterior e na goteira parietocólica direita. Na região do sigmóide, observava-se acentuada redução do calibre da alça e dilatação gasosa a montante.

$\mathrm{O}$ paciente foi encaminhado à cirurgia, sendo realizada colostomia para descompressão intestinal, evidenciando-se, no ato cirúrgico, ruptura do ceco, com lacerações da serosa e coleção sanguínea na cavidade. $\mathrm{O}$ paciente faleceu no pósoperatório tardio.

\section{DISCUSSÃO}

Obstrução em alça fechada ocorre quando duas extremidades de alça permanecem ocluídas, como nas torções ou vólvulos. Na obstrução do cólon há um processo obstrutivo em alça distal, com válvula ileocecal competente ${ }^{(1,2)}$.

Estenose do cólon é oito vezes mais freqüente no lado esquerdo que no direito. No paciente idoso, a causa mais freqüente de obstrução são os carcinomas de reto e sigmóide, ambos por serem do tipo anular e estenosante ${ }^{(\mathbf{1}, \mathbf{3})}$. Como as paredes do cólon ascendente e do ceco são mais finas, estes segmentos sofrem distensão muito mais acentuada do que a porção pós-estenótica no sigmóide ou no reto ${ }^{(11,12)}$.

$\mathrm{O}$ ceco distendido adquire aspecto ovalar e liso, com grande nível líquido no seu interior, e em alguns casos ocorre também distensão gasosa do apêndi$\mathrm{ce}^{(\mathbf{1 , 1 2})}$. A redução do calibre da alça produz uma barreira física que impede a progressão do conteúdo intestinal, acarretando aumento da pressão intraluminal e dificuldade do retorno venoso, o que determina edema da parede e transudação de plasma para o peritônio e para a luz intestinal. Com a permanência do processo, ocorre o estrangulamento da alça por interrupção do fluxo sanguíneo, necrose e perfuração ${ }^{(3,11)}$.

Os sinais e sintomas da obstrução mecânica são dor abdominal, vômitos, constipação, dispnéia, fraqueza muscular e desidratação. A fraqueza muscular decorre da depleção de potássio, a dispnéia reflete a redução dos movimentos diafragmáticos devido à distensão abdominal, e a desidratação decorre tanto da perda de líquidos e eletrólitos pelo vômito como pela grande quantidade de líquido extravasado para a luz intestinal e peritônio. A distensão intestinal mantida provoca aumento de secreção intraluminal, contaminada por sangue e bactérias, que, uma vez reabsorvida, determina bacteremia e choque séptico ${ }^{(11)}$. A ruptura dos segmentos retroperitoneais do cólon determina extravasamento de líquido e sangue também para o espaço pararrenal anterior ${ }^{(\mathbf{1 2})}$.

O exame radiológico deve sempre ser complementado pela tomografia computadorizada, com a finalidade de pesquisar a extensão serosa e peritoneal da neoplasia, assim como para seu estadiamento, que depende da presença ou não de implantes metastáticos a distância.

A tomografia computadorizada vemse firmando cada vez mais como método de diagnóstico eficaz no exame do trato gastrointestinal. Quando existe obstrução intestinal mecânica associada com massa de tecidos moles, ela tem papel importante para descartar a possibilidade de perfuração de alça, que muitas vezes passa despercebida ao exame radiológico com meio de contraste barita- do. Também no abdome agudo obstrutivo de alça intestinal ela consegue detectar as alterações patológicas decorrentes da perfuração, possibilitando avaliação, tanto da luz como dos tecidos adjacentes.

\section{REFERÊNCIAS}

1. Frimann-Dahl J. Mechanical obstruction of the large bowel. In: Frimann-Dahl J, ed. Roentgen examinations in acute abdominal diseases. Springfield: Charles C. Thomas, 1960:199-210.

2. Frimann-Dahl J. The acute abdomen. In: FrimannDahl J, ed. Alimentary tract roentgenology. London: Mosby, 1967:155-68.

3. Coelho RB, Raso HM. Obstrução intestinal. In: Bogliolo L, ed. Patologia. Rio de Janeiro: Guanabara Koogan, 1976:583-9.

4. Binkert CA, Ledermann H, Jost R, Saurenmann P, Decurtis M, Zollikofer CL. Acute colonic obstruction: clinical aspects and cost-effectiveness of preoperative and palliative treatment with selfexpanding metallic stents - a preliminary report. Radiology 1998;206:199-204.

5. De Gregório MA, Mainar A, Tejero E, et al. Acute colorectal obstruction: stent placement for palliative treatment - results of a multicenter study. Radiology 1998;209:117-20.

6. Choo IW, Do YS, Suh SW, et al. Malignant colorectal obstruction: treatment with a flexible covered stent. Radiology 1998;206:415-21.

7. Irvin GL, Horsley JS, Caruana JA Jr. The morbidity and mortality of emergent operations for colorectal disease. Ann Surg 1984;199:598-603.

8. Scott NA, Jeacock J, Kingston RD. Risk factors in patients presenting as an emergency with colorectal cancer. Br J Surg 1995;82:321-3.

9. Soonawalla Z, Thakur K, Boorman P, Macfarlane P, Sathananthan N, Parker M. Use of self-expanding metallic stent in the management of obstruction of the sigmoid colon. AJR 1998;171:633-6.

10. Megibow AJ. The gastrointestinal tract. In: Haaga JR, Lanzieri CF, Zerhouni EA, eds. Computed tomography and resonance imaging of the whole body. St. Louis, Washington, Toronto: Mosby, 1994:873-4.

11. Mendeloff AI, Seligman AM. Obstrução intestinal aguda: In: Harrison's Principles of internal medicine. Rio de Janeiro: Guanabara Koogan, 1970: 1356-7.

12. Mayers MA. Extraperitoneal spaces: normal and pathologic anatomy. In: Mayers MA, ed. Dynamic radiology of the abdomen. Normal and pathologic anatomy. Berlin: Springer-Verlag, 1976:123-9.

13. Canon CL, Baron TH, Morgan DE, Dean PA, Koehler RE. Treatment of colonic obstruction with expandable metal stents: radiologic features. AJR 1997;168:199-205. 